\title{
openheart Real-world presentation with heart failure in primary care: do patients selected to follow diagnostic and management guidelines have better outcomes?
}

\author{
Alex Bottle, ${ }^{1}$ Dani Kim, ${ }^{1}$ Paul P Aylin, ${ }^{1}$ F Azeem Majeed, ${ }^{2}$ Martin R Cowie, ${ }^{3}$ \\ Benedict Hayhoe ${ }^{2}$
}

\begin{abstract}
- Additional material is published online only. To view please visit the journal online (http://dx.doi.org/10.1136/ openhrt-2018-000935)
\end{abstract}

To cite: Bottle A, Kim D, Aylin PP, et al. Real-world presentation with heart failure in primary care: do patients selected to follow diagnostic and management guidelines have better outcomes?. Open Heart 2018;5:e000935. doi:10.1136/ openhrt-2018-000935

Received 12 September 2018 Revised 15 October 2018 Accepted 17 October 2018

Check for updates

(c) Author(s) (or their employer(s)) 2018. Re-use permitted under CC BY-NC. No commercial re-use. See rights and permissions. Published by BMJ.

${ }^{1}$ Dr Foster Unit, Department of Primary Care and Public Health, Imperial College London, London, UK

${ }^{2}$ Department of Primary Care and Public Health, Imperial College London, London, UK ${ }^{3}$ National Heart \& Lung Institute, Royal Brompton Hospital, Imperial College London, Chelsea, UK

Correspondence to Dr Alex Bottle; robert.bottle@ imperial.ac.uk

\section{ABSTRACT}

Objective To describe associations between initial management of people presenting with heart failure (HF) symptoms in primary care, including compliance with the recommendations of the National Institute for Health and Care Excellence (NICE), and subsequent unplanned hospitalisation for HF and death.

Methods This is a retrospective cohort study using data from general practices submitting records to the Clinical Practice Research Datalink. The cohort comprised patients diagnosed with HF during 2010-2013 and presenting to their general practitioners with breathlessness, fatigue or ankle swelling.

Results 13897 patients were included in the study. Within the first 6 months, only $7 \%$ had completed the NICE-recommended pathway; another $18.6 \%$ had followed part of it (B-type natriuretic peptide testing and/ or echocardiography, or specialist referral). Significant differences in hazards were seen in unadjusted analysis in favour of full or partial completion of the NICErecommended pathway. Covariate adjustment attenuated the relations with death much more than those for HF admission. Compared with patients placed on the NICE pathway, treatment with HF medications had an HR of 1.16 $(95 \% \mathrm{Cl} 1.05$ to $1.28, \mathrm{p}=0.003)$ for $\mathrm{HF}$ admission and 1.03 $(95 \% \mathrm{Cl} 0.90$ to $1.17, \mathrm{p}=0.674)$ for death. Patients who partially followed the NICE pathway had similar hazards to those who completed it. Patients on no pathway had the highest hazard for HF admission at 1.30 (95\% 1.18 to $1.43, p<0.001$ ) but similar hazard for death.

Conclusions Patients not put on at least some elements of the NICE-recommended pathway had significantly higher risk of $\mathrm{HF}$ admission but non-significant higher risk of death than other patients had.

\section{INTRODUCTION}

Around 40 million people have heart failure (HF) worldwide, ${ }^{1}$ with 550000 in the $\mathrm{UK}^{2}$ Timely diagnosis is necessary to allow initiation of appropriate management, ${ }^{34}$ but this can be difficult, and with no single diagnostic screening test professionals traditionally rely
Key messages

What is already known on this subject?

- Early identification of heart failure (HF) is key to effective management, but diagnosis can be challenging, especially in primary care.

- National Institute for Health and Care Excellence (NICE) guidelines recommend investigation of patients with suspected HF with echocardiogram and/ or serum natriuretic peptide, followed by specialist referral; the degree of adherence to this recommendation is low, but the relation with outcomes in practice is not known.

What this study adds?

- Of patients with heart failure (HF) symptoms recorded in primary care, those who followed a pathway aligned with the NICE guidelines had significantly lower risk of emergency HF admission than, but similar risk of death to, patients treated symptomatically or managed conservatively in the risk-adjusted models.

How might this impact on clinical practice?

- There may be missed opportunities for earlier HF diagnosis in primary care, and more patients could benefit from better outcomes if investigated for HF rather than treated symptomatically or managed conservatively.

on the classic symptoms of breathlessness, ankle swelling and fatigue to alert them to the possibility of the diagnosis. ${ }^{5}$ Patients with HF often present first in primary care where diagnosis and initial management are supported by professional guidelines. ${ }^{5-8}$ The National Institute for Health and Care Excellence (NICE) provides recommendations on the use of serum B-type natriuretic peptide (NP) or N-terminal pro-B-type natriuretic peptide testing for patients with symptoms suggestive of HF, as well as echocardiographic imaging 
of the heart ('echo'), specialist referral, and prescription of medications shown to improve symptoms and prognosis in HF with a reduced ejection fraction (HFrEF) (beta-blockers, ACE inhibitors or angiotensin II blockers (ARB), and mineralocorticoid receptor antagonists). ${ }^{6}$

We have previously described ${ }^{9}$ the different diagnostic and management approaches ('pathways') in primary care in patients in England subsequently diagnosed with HF. In this study, we use the same linked primary and secondary care data to describe associations between general practitioner (GP) initial diagnostic work-up and management pathway and subsequent emergency hospitalisation for HF or death.

\section{METHODS}

\section{Data sources}

The Clinical Practice Research Datalink (CPRD) contains anonymised electronic records from about $7 \%$ of UK general practices from 1987 to the present. Primary care records are linked nationally to hospital admissions (Hospital Episode Statistics (HES) ) and the death registry (Office for National Statistics (ONS)). We used data for the 10-year period from 2005 to 2014.

\section{Patient cohort}

We searched for patients diagnosed with HF between 1 January 2010 and 31 March 2013. Diagnosis date was defined as the first coded record of HF either in the primary care record or in hospital admission data in that period; patients with any HF code in the previous 5 years were excluded. In primary care records, we identified consultations using published codes augmented by our local clinicians (see online supplementary appendix $\left.1^{9}{ }^{10}\right)$. In HES, the International Statistical Classification of Diseases, 10th revision, codes could be recorded as either primary or secondary diagnoses; the earliest record of either was used.

Seeking to analyse the effect of management received after presentation with symptom(s), we included only patients with a record of HF symptom(s) before their diagnosis (tracking back up to 5 years). Published clinical codes for tests, medications and referrals were used (see online supplementary appendix 1) to identify the management between the time of first presentation and diagnosis.

The following patient characteristics were defined at the time of their first symptom(s): gender, age, deprivation quintile (using the Index of Multiple Deprivation score 2010), history of selected comorbidities (tracking back up to 5 years) and symptom type at first presentation (breathlessness only, ankle swelling only, fatigue only, or two or more symptoms). We also recorded whether patients had had another consultation for HF symptoms within 6 months of first presentation. Comorbidities from the Charlson code set were defined as per Khan et $a l,{ }^{11}$ with some additions by our team. ${ }^{9}$ Otherwise, codes were identified using the CPRD medical and product dictionaries.

\section{Outcomes}

Outcomes of interest were first-ever hospital admission for $\mathrm{HF}$ and all-cause death. For patients with HF recorded first in primary care, the former was defined as an admission with HF as primary diagnosis; for patients with HF recorded first in hospital, it was defined as an admission with HF recorded as primary or secondary diagnosis. Death was identified via the ONS mortality records.

\section{Pathway definitions}

We classified management into four 'pathways', combining some of the seven in our earlier study, ${ }^{9}$ depending on investigations, medications and referrals recorded in primary care consultations using two time frames: the first 6 months and 5 years following $\mathrm{HF}$ symptom. Pathway 1 (the 'NICE-recommended pathway') comprised patients investigated in accordance with the NICE recommendations (serum NP test and/or echocardiogram and referral to a specialist). Pathway 2 referred to partial concordance with guideline recommendations. Patients who had no investigations or referral recorded but were either already taking or started on relevant medications for HF (ACE inhibitors, ARBs and HF-specific beta-blockers prescribed between 1 year before and after the date of HF symptom) were categorised as pathway 3. Pathway 4 ('Other pathway') covered those who had received no NICE-recommended diagnostic or treatment elements, although they may have received other relevant management options, such as smoking cessation advice, influenza vaccination, an ECG and/or a non-HF-specific beta-blocker.

\section{Time frame, exclusion criteria and sensitivity analyses}

Standard CPRD exclusion criteria were applied first (see online supplementary appendix 1). Patients with any HF code in the 5 years before January 2010 were also excluded.

Few patients completed the NICE elements within its recommended time frame of at most 6 weeks. To accommodate this, we allowed first 6 months and second 5 years after their first symptom presentation for all the elements to be done, allocating each patient to a particular pathway according to how they had been managed in these time periods. Some patients deteriorate quickly following presentation with symptoms, and hospitalisation for HF and death both interrupt the GP's management and completion of the NICE pathway (immortal time bias). To avoid this interruption, we excluded those who died or were hospitalised for HF within 6 months of their first symptom. In sensitivity analyses, we compared the proportions on each pathway and the crude outcome rates for the excluded patients with the included ones and with all patients combined. We also modelled all patients without exclusions for outcome by incorporating the pathway as a time-varying covariate. 


\section{Statistical analysis}

Patient characteristics were summarised for all patients at the time of first recorded HF symptom. Cumulative incidence functions described the crude association between pathway taken and each outcome. Cause-specific hazards regression models were used to calculate adjusted HRs and 95\% CIs for the association between pathway and outcome; these hazards account for the competing risk of death in the model for first-ever HF admission. Models also included gender, age, source of HF diagnosis (whether primary care or hospital—only for death), comorbidity history (as count and as individual diseases), symptom at first presentation, and whether patients received another consultation for HF symptoms within 6 months of their first one. The proportional hazards assumption was checked by plotting the Schoenfeld residuals against time. Analyses were conducted using SAS V.9.4.

\section{RESULTS}

There were 2610 patients excluded because they died or were hospitalised for HF within 6 months of symptom presentation, leaving 13897 patients for analysis (table 1). The included patients were typically elderly with multiple comorbidities. The majority $(65 \%)$ had breathlessness recorded as their presenting symptom, and about one in four presented more than once in primary care in the first 6 months with an HF symptom. Table 2 summarises how they were managed in the first 6 months and 5 years after initial presentation. Within the first 6 months, only $7 \%$ had completed the NICE-recommended pathway (pathway 1); another 18.6\% followed part of it (pathway 2). After 5 years, only $25 \%$ had completed the NICE-recommended pathway. In the excluded patients, $7 \%$ completed pathway 1 within 6 months, same as for the included ones, but fewer (23\%) were on no pathway.

Table 3 gives the crude outcome rates from presentation with symptoms to the end of study follow-up. The rates of both outcomes were lowest for patients on the NICE-recommended pathway (second column)—advantages that were maintained even without excluding any of the patients who had an outcome within 6 months of presentation with symptoms (last column).

Figures 1 and 2 show the crude rate of each outcome over time by pathway. There were statistically significant differences between pathways, although with some change in the differences between pathways over the duration of follow-up. Those on the NICE-recommended pathway (pathway 1) initially had a higher rate of $\mathrm{HF}$ admission, but by the end of the follow-up period those with partial NICE adherence (pathway 2) or no pathway (pathway 4) had the highest admission rate. Those following no pathway also had the highest rate of death by the end of follow-up.

In the unadjusted comparisons, only deprivation and some comorbidities were not significant predictors of outcomes (see online supplementary appendix 1 ).
Table 1 Patient characteristics at symptom recording ( $\mathrm{N}=13$ 897)

\begin{tabular}{|c|c|c|c|}
\hline Variable & Level & $\mathbf{n}$ & $\%$ \\
\hline \multirow[t]{2}{*}{ Gender } & Male & 6672 & 48.0 \\
\hline & Female & 7225 & 52.0 \\
\hline \multirow[t]{5}{*}{ Age group } & $<45$ & 234 & 1.7 \\
\hline & $45-64$ & 2052 & 14.8 \\
\hline & $65-74$ & 3422 & 24.6 \\
\hline & $75-84$ & 5401 & 38.9 \\
\hline & $85+$ & 2788 & 20.1 \\
\hline \multirow[t]{5}{*}{ IMD quintile } & 1 (least deprived) & 2731 & 19.7 \\
\hline & 2 & 3233 & 23.3 \\
\hline & 3 (average) & 2931 & 21.1 \\
\hline & 4 & 2631 & 18.9 \\
\hline & 5 (most deprived) & 2371 & 17.1 \\
\hline \multirow[t]{2}{*}{ Diagnosis source of HF } & Primary care & 3388 & 24.4 \\
\hline & Hospital & 10509 & 75.6 \\
\hline \multirow[t]{12}{*}{ Comorbidity } & Atrial fibrillation & 2603 & 18.7 \\
\hline & Other arrhythmias & 1349 & 9.7 \\
\hline & Myocardial infarction & 667 & 4.8 \\
\hline & Coronary artery disease & 3344 & 24.1 \\
\hline & Myocarditis & 70 & 0.5 \\
\hline & Hypertension & 8150 & 58.6 \\
\hline & Stroke & 843 & 6.1 \\
\hline & Diabetes & 2727 & 19.6 \\
\hline & Congenital heart disease & 60 & 0.4 \\
\hline & Chronic pulmonary disease & 3685 & 26.5 \\
\hline & Peripheral vascular disease & 1018 & 7.3 \\
\hline & Renal disease & 3264 & 23.5 \\
\hline \multirow{5}{*}{$\begin{array}{l}\text { Number of } \\
\text { comorbidities }\end{array}$} & 0 & 2252 & 16.2 \\
\hline & 1 & 3561 & 25.6 \\
\hline & 2 & 3419 & 24.6 \\
\hline & 3 & 2452 & 17.6 \\
\hline & $4+$ & 2213 & 15.9 \\
\hline \multirow[t]{4}{*}{ Symptom type } & Breathlessness only & 9096 & 65.5 \\
\hline & Ankle swelling only & 2251 & 16.2 \\
\hline & Fatigue only & 2355 & 16.9 \\
\hline & Two or more symptoms & 195 & 1.4 \\
\hline $\begin{array}{l}\text { Consultation for HF } \\
\text { symptom within } \\
6 \text { months of first } \\
\text { symptom }\end{array}$ & Once or more & 3793 & 27.3 \\
\hline
\end{tabular}

$H F$, heart failure; IMD, Index of Multiple Deprivation.

Strong differences in unadjusted hazards were identified by pathway for both outcomes. For example, compared with the NICE-recommended pathway (pathway 1), those on no pathway (pathway 4) had an HR of 1.28 for the first HF admission $(p<0.001)$ and 1.20 for death $(p<0.001)$. 
Table 2 Management pathway taken within 6 months or 5 years of first presentation with HF symptom

\begin{tabular}{|c|c|c|c|c|}
\hline \multirow[b]{2}{*}{ Pathway } & \multicolumn{2}{|c|}{ Within 6 months of symptom } & \multicolumn{2}{|c|}{ Within 5 years of symptom } \\
\hline & $\mathbf{n}$ & $\%$ & $\mathbf{n}$ & $\%$ \\
\hline 1. NICE recommendation (echo/NP and referral) & 976 & 7.0 & 3409 & 24.5 \\
\hline 2. Guidance partially followed (echo/NP or referral) & 2589 & 18.6 & 4657 & 33.5 \\
\hline 3. Treatment only-HF medications but no NP/echo or referral & 5117 & 36.8 & 3938 & 28.3 \\
\hline 4. No NP/echo, referral or HF medications & 5215 & 37.5 & 1893 & 13.6 \\
\hline Total & 13897 & - & 13897 & - \\
\hline
\end{tabular}

HF, heart failure; NICE, National Institute for Health and Care Excellence; NP, natriuretic peptide.

Following covariate adjustment, the HR for those on no pathway was only slightly changed for the first HF admission $(1.30, \mathrm{p}<0.001)$ but attenuated to $1.11(\mathrm{p}=0.123)$ for death (table 4). The main factor responsible for the attenuation was adjustment for place of diagnosis (primary care or hospital): patients diagnosed during admission for HF had higher hazard of death $(\mathrm{HR}=1.34, \mathrm{p}<0.001)$. Only small differences were seen between crude and adjusted hazards of death for the other pathways.

We tested the proportional hazards assumption for each predictor. Despite the impression given by figures 1 and 2 of the crude associations, where the NICE pathway patients initially had higher HF admission rates, the assumption was met for all predictors, including pathway, in the full model (see online supplementary appendix 1 for crude outcome rates at 1 year).

The results presented relate to the association between outcome and pathway taken within the first 6 months of symptoms. The same analyses were carried out for the pathway taken within 5 years but are not shown as the effects were similar but simply greater in magnitude. The models without exclusions for outcome but with pathway as a time-varying covariate led to similar conclusions but attenuated HRs for emergency HF admission for treatment with medication (HR=1.06 (0.99 to 1.13), $\mathrm{p}=0.077)$ and for no pathway (HR=1.14 (1.07 to 1.21), $\mathrm{p}<0.001$; see online supplementary appendix 1$)$.

\section{DISCUSSION}

We found strong crude and covariate-adjusted associations between initial management pathway and the first emergency admission for HF. The $7 \%$ of patients who followed the NICE-recommended route had the lowest hazards for HF admission; those treated with medication but not investigated or referred had $16 \%$ higher odds of HF admission, and those on no pathway had the highest odds. Crude associations between initial management pathway and death became statistically non-significant after covariate adjustment.

\section{Comparisons with other studies}

CPRD was recently used to look at prognosis following HF diagnosis in primary care and secondary outpatient care, ${ }^{4}$ focusing on place of diagnosis (either primary or secondary care) rather than the route to diagnosis. Patients admitted to hospital with worsening HF but not known in primary care to have HF had the worst prognosis and management: we excluded these patients from

\begin{tabular}{|c|c|c|c|}
\hline Pathway & Included, $\mathrm{n}(\%)^{*}$ & Excluded, $n(\%)$ & Total, n (\%) \\
\hline \multicolumn{4}{|l|}{ Emergency admission for HF } \\
\hline 1. NICE recommendation (echo/NP and referral) & $558(57.2)$ & $175(96.2)$ & $727(62.8)$ \\
\hline 2. Guidance partially followed (echo/NP or referral) & $1810(69.9)$ & $627(96.3)$ & $2361(72.9)$ \\
\hline 3. Treatment only-HF medications but no NP/echo or referral & $4153(81.2)$ & $1150(97.6)$ & $5002(79.5)$ \\
\hline 4. No NP/echo, referral or HF medications & $4410(84.6)$ & $572(95.5)$ & $4881(84.0)$ \\
\hline Total HF admissions (overall rate as \%) & $10931(78.7)$ & $2525(96.7)$ & $12971(78.6)$ \\
\hline \multicolumn{4}{|l|}{ Death } \\
\hline 1. NICE recommendation (echo/NP and referral) & $280(28.7)$ & $64(35.2)$ & $344(29.7)$ \\
\hline 2. Guidance partially followed (echo/NP or referral) & $914(35.3)$ & $253(38.9)$ & $1167(36.0)$ \\
\hline 3. Treatment only-HF medications but no NP/echo or referral & $2106(41.2)$ & $536(45.5)$ & $2642(42.0)$ \\
\hline 4. No NP/echo, referral or HF medications & $2287(43.9)$ & $366(61.1)$ & $2653(45.6)$ \\
\hline Total deaths (overall rate as \%) & $5587(40.2)$ & $1219(46.7)$ & $6806(41.2)$ \\
\hline
\end{tabular}

${ }^{*} \chi^{2}$ tests for trend across pathways: $p<0.001$ for both outcomes.

$\mathrm{HF}$, heart failure; NICE, National Institute for Health and Care Excellence; NP, natriuretic peptide. 


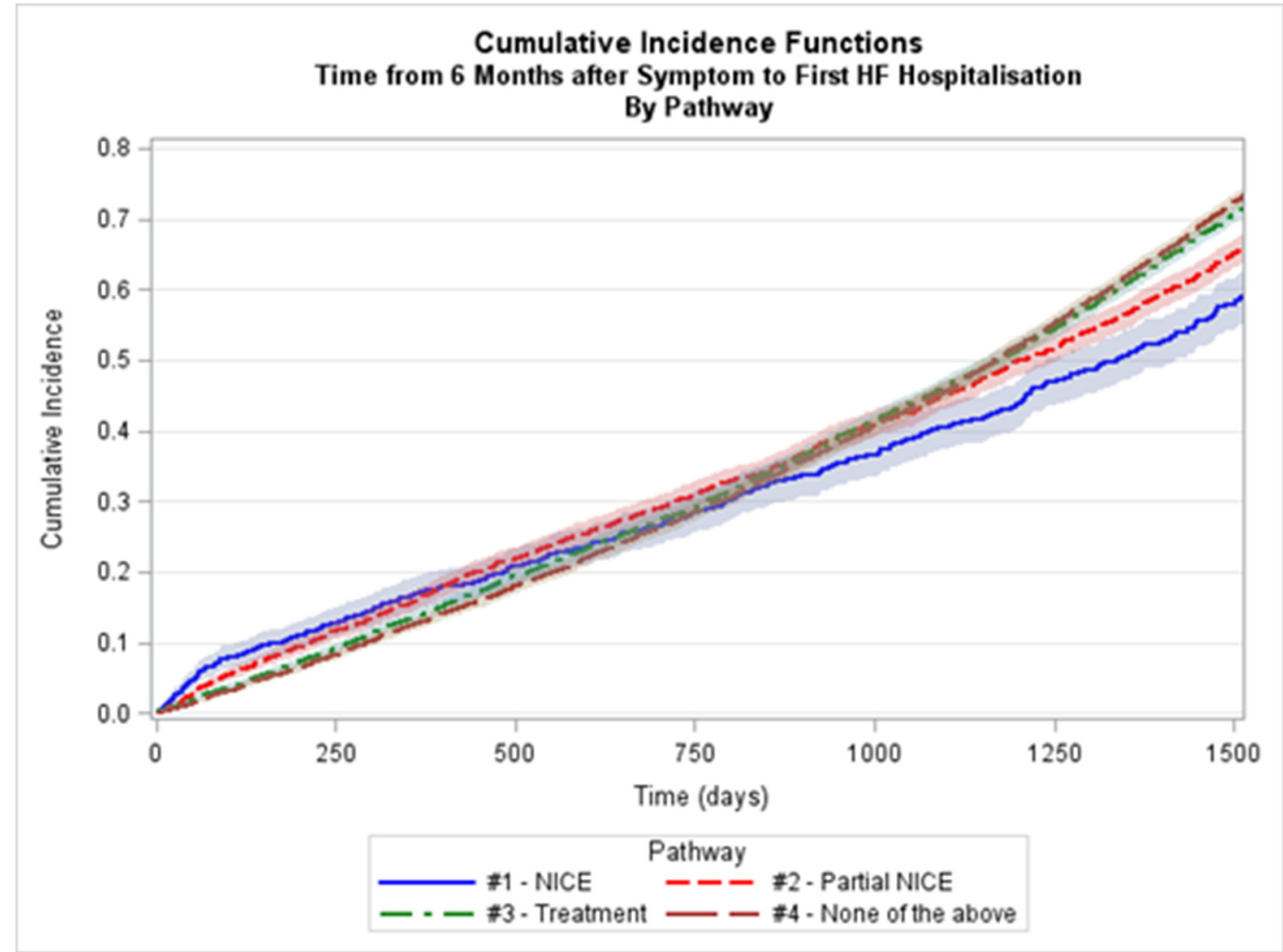

Figure 1 Cumulative incidence of, and $95 \% \mathrm{Cl}$ for, first HF emergency admission starting from 6 months after symptom by pathway taken $(p<0.0001)$. HF, heart failure; NICE, National Institute for Health and Care Excellence.

our study by requiring at least 6 months between presentation with symptoms and either outcome.

\section{Strengths and limitations}

The CPRD population is broadly representative of the UK population in terms of age, sex and ethnicity, ${ }^{12}$ and the database is well used in research ${ }^{13}$; HES covers all National Health Service (NHS) hospitals in England and has highly accurate primary diagnosis coding. ${ }^{14}$ Linkage between them and with the death registry allows tracking of patient pathways through the whole system. CPRD data are derived from clinical codes entered by GPs during consultations and therefore reflect information that GPs have for decision-making. In the UK, the national pay-for-performance scheme, the Quality and Outcomes Framework, incentivises the recording of clinical codes for certain chronic diseases including HF. This makes proper diagnoses when known more likely to be used than, for example, symptom codes. ${ }^{15}$

However, clinical coding in primary care remains highly variable, ${ }^{16}$ with much important data recorded in free text. We defined our cohort based on HF symptoms recorded in GP consultations. The validity of this depends both on patients reporting symptoms and on GPs recording them as Read codes; we note that we cannot be certain that the first recording of any of the three symptoms or treatment with medication marked the onset of HF. In our previous study, we found a small proportion of patients following the NICE-recommended HF pathway despite having none of the three main symptoms entered. ${ }^{9}$ It seems likely that there is particular variability in coding of presenting symptoms, as opposed to diagnostic or examination codes which may be subject to incentives. This may explain, for example, why we found no association between reconsulting for HF symptoms and outcomes; coding of repeat occurrences of the same symptom may be particularly unreliable. We have described elsewhere other data quality considerations, such as CPRD lacking ejection fraction and NP levels in most patients. ${ }^{9}$ Similar issues apply to coding investigations such as echocardiography.

We allowed 6 months from first presentation with HF symptom(s) for the GP to suspect HF and complete the pathway. Suspicion may develop slowly if the main symptom is fatigue and/or the patient already has other conditions such as COPD associated with similar symptoms. However, extending this period would result in loss of cases and changes in assigned pathway for an increasing number of patients. We were encouraged by the between-pathway differences in crude outcome rates in the included set of patients being similar to those for all patients with no exclusion (table 3).

In an observational study, we cannot draw causal inferences from the associations between pathway and 


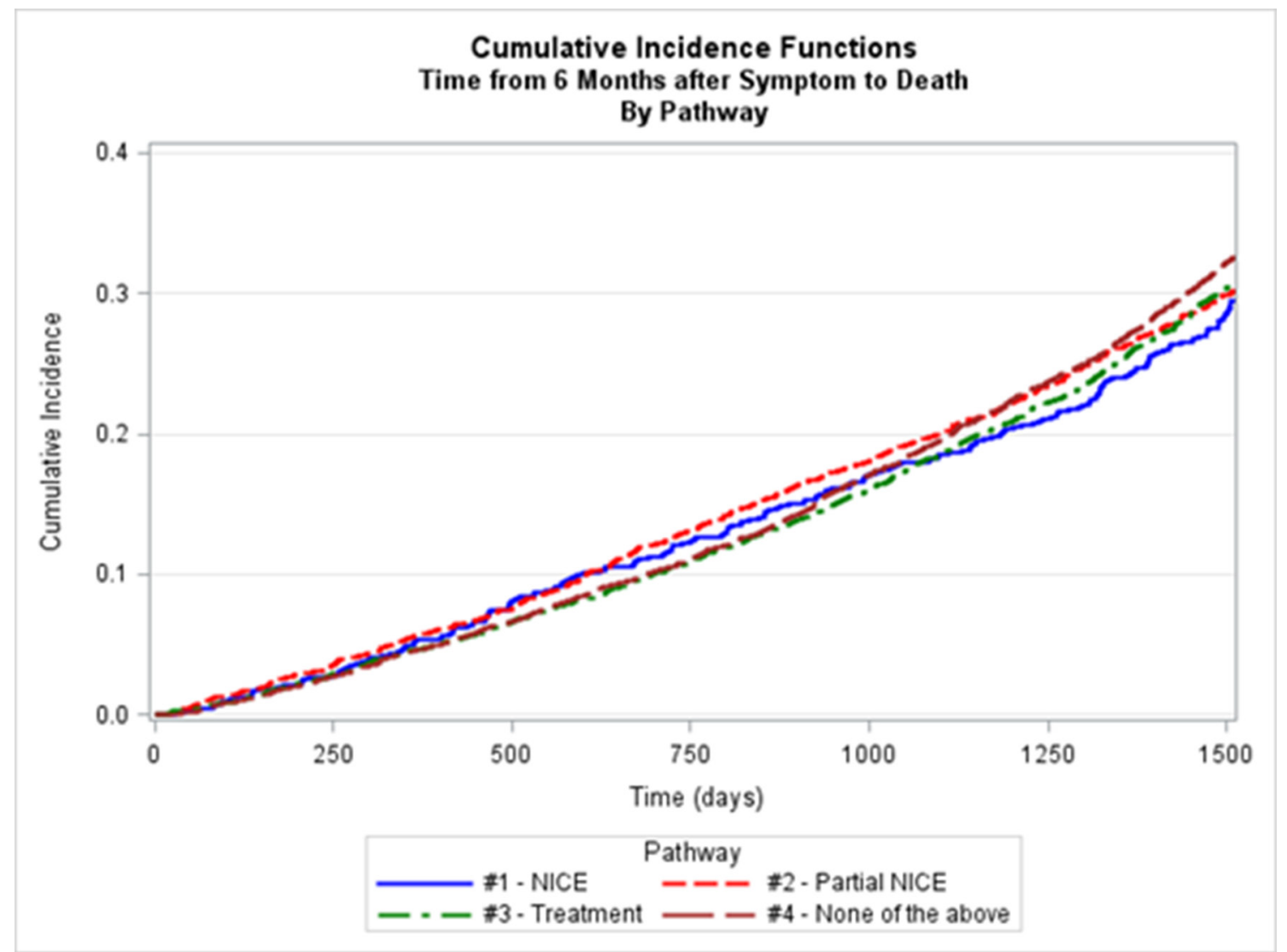

Figure 2 Cumulative incidence of death starting from 6 months after symptom by pathway taken $(p<0.0001)$. Confidence bands are not shown. NICE, National Institute for Health and Care Excellence.

outcomes. Our models adjusted for several confounders, and the elevated crude HRs for death were notably attenuated. Adjustment had much less impact on the HRs for the first HF admission. Ejection fraction varies by age and sex, so adjustment for these variables will represent partial adjustment for ejection fraction, ${ }^{17}$ but we cannot tell whether adjustment for unavailable factors such as ejection fraction and NP levels would have modified these relationships. We can say, however, that patients who avoid admission or death for at least 6 months from initial presentation with symptom(s) and who follow the NICE-recommended pathway have significantly lower odds of HF admission than others.

More than one in three of our sample were given medication for HF without referral for echocardiography. These patients, and those following no pathway, had higher risk of admission for HF risk than those following the NICE-recommended pathway. Overall, it is likely that up to half of people with HF have a preserved left ventricular ejection fraction (HFpEF) and therefore will not likely benefit prognostically from drug treatment ${ }^{17}$; those following a NICE pathway may at least be offered cardiac rehabilitation following cardiology review, which can benefit those with HFpEF, ${ }^{18}$ and are likely to have better controlled symptoms and comorbidity. Even if patients offered only treatment (without proper diagnosis) had HFrEF and could therefore benefit, this may not have been properly implemented: HF medications need to be titrated up over time, which may not occur without specialist clinic instructions. Furthermore, as our group of 'HF medications' is not specific to HF, these patients might have been given treatments (eg, an ACE inhibitor) for another indication, such as hypertension or diabetes. Those on neither a NICE-recommended pathway nor treatment had the highest risk of admission. It seems likely that some of these were more unwell than those on the NICE pathway (GPs may have sent some of those on pathways 2, 3 and 4 straight to the hospital if acutely unwell), and some will have HFrEF and potentially amenable to treatment which they did not receive.

Both referrals and echocardiograms may be made for reasons other than suspected HF. Nevertheless, we have assumed that in our cohort, consisting of patients with a recorded diagnosis of $\mathrm{HF}$, they were made for this indication. Serum NP testing was recommended in the 2005 European Society of Cardiology and the August 2010 NICE guidance but only available for a minority of practices during our study period. It is now more widely used, although threshold values are still under discussion. ${ }^{3}$

Our study relates to a centrally funded healthcare system in a large European country with detailed data collection available for analysis, but timely diagnosis and management of HF in primary care is not a problem unique to the UK. ${ }^{19} 20$ We focused on the UK's NICE 
Table 4 Adjusted HRs for the first HF emergency admission and death since time from 6 months after symptom (using comorbidity history as individual diseases)

\begin{tabular}{|c|c|c|c|c|}
\hline \multirow[b]{2}{*}{ Variable } & \multicolumn{2}{|c|}{$\begin{array}{l}\text { First HF emergency } \\
\text { admission }\end{array}$} & \multicolumn{2}{|l|}{ Death } \\
\hline & HR $(95 \%$ Cl) & $P$ values & HR (95\% Cl) & $P$ values \\
\hline \multicolumn{5}{|l|}{ Gender } \\
\hline Male vs female & 0.98 (0.95 to 1.02$)$ & 0.420 & 1.19 (1.13 to 1.26$)$ & $<0.001$ \\
\hline \multicolumn{5}{|l|}{ Age at symptom } \\
\hline Per 5-year increase & 1.05 (1.04 to 1.07 ) & $<0.001$ & 1.24 (1.22 to 1.26$)$ & $<0.001$ \\
\hline \multicolumn{5}{|l|}{ HF diagnosis source } \\
\hline Hospital vs primary care & NA & NA & 1.39 (1.29 to 1.49$)$ & $<0.001$ \\
\hline IMD quintile & & 0.003 & & 0.037 \\
\hline 2 vs 1 & 1.04 (0.98 to 1.10$)$ & 0.212 & 1.01 (0.93 to 1.09$)$ & 0.842 \\
\hline 3 vs 1 & 1.03 (0.97 to 1.10$)$ & 0.307 & 0.97 (0.89 to 1.05$)$ & 0.446 \\
\hline 4 vs 1 & 1.09 (1.02 to 1.16$)$ & 0.010 & 1.02 (0.94 to 1.11$)$ & 0.654 \\
\hline 5 vs 1 & $1.12(1.05$ to 1.20$)$ & $<0.001$ & 1.11 (1.02 to 1.22$)$ & 0.020 \\
\hline \multicolumn{5}{|l|}{ Comorbidity } \\
\hline Atrial fibrillation & 1.10 (1.04 to 1.16$)$ & $<0.001$ & $1.12(1.05$ to 1.21$)$ & 0.001 \\
\hline Other arrhythmias & 1.00 (0.94 to 1.08$)$ & 0.897 & 0.97 (0.88 to 1.07$)$ & 0.512 \\
\hline Myocardial infarction & 0.99 (0.90 to 1.08$)$ & 0.766 & 1.15 (1.01 to 1.31$)$ & 0.041 \\
\hline Coronary artery disease & 0.99 (0.94 to 1.04$)$ & 0.657 & 0.86 (0.80 to 0.92$)$ & $<0.001$ \\
\hline Myocarditis & 1.33 (0.98 to 1.81$)$ & 0.066 & 0.89 (0.59 to 1.35$)$ & 0.582 \\
\hline Hypertension & 1.03 (0.99 to 1.08$)$ & 0.136 & 0.90 (0.85 to 0.96$)$ & $<0.001$ \\
\hline Stroke & 1.030 .95 to 1.11 & 0.478 & 1.11 (1.00 to 1.24$)$ & 0.050 \\
\hline Diabetes & 1.12 (1.07 to 1.18$)$ & $<0.001$ & 1.09 (1.02 to 1.17$)$ & 0.017 \\
\hline Congenital heart disease & 1.27 (0.91 to 1.78$)$ & 0.167 & 1.26 (0.81 to 1.97$)$ & 0.309 \\
\hline Chronic pulmonary disease & 1.09 (1.04 to 1.14$)$ & $<0.001$ & 1.15 (1.08 to 1.22$)$ & $<0.001$ \\
\hline Peripheral vascular disease & 1.21 (1.12 to 1.30$)$ & $<0.001$ & 1.36 (1.23 to 1.50$)$ & $<0.001$ \\
\hline Renal disease & 1.18 (1.13 to 1.24$)$ & $<0.001$ & 1.15 (1.08 to 1.23$)$ & $<0.001$ \\
\hline Pathway & & $<0.001$ & & 0.071 \\
\hline 1. (NICE-recommended) & 1 & & 1 & \\
\hline 2. Guidance partially followed vs 1 & 1.11 (1.00 to 1.23$)$ & 0.052 & 1.03 (0.89 to 1.18$)$ & 0.700 \\
\hline 3. Treatment only—HF medications but no NP/echo or referral vs 1 & 1.16 (1.05 to 1.28$)$ & 0.003 & $1.03(0.90$ to 1.17$)$ & 0.674 \\
\hline 4. No NP/echo, referral or HF medications vs 1 & 1.30 (1.18 to 1.43$)$ & $<0.001$ & $1.11(0.97$ to 1.27$)$ & 0.123 \\
\hline Symptom type & & $<0.001$ & & $<0.001$ \\
\hline Breathlessness only vs fatigue only & 1.06 (1.01 to 1.12 ) & 0.027 & 1.19 (1.10 to 1.29$)$ & $<0.001$ \\
\hline Ankle swelling only vs fatigue only & 1.17 (1.10 to 1.25$)$ & $<0.001$ & 1.09 (0.99 to 1.20$)$ & 0.066 \\
\hline Two or more symptoms vs fatigue only & 0.70 (0.60 to 0.82$)$ & $<0.001$ & 0.96 (0.76 to 1.22$)$ & 0.760 \\
\hline \multicolumn{5}{|l|}{ Further consultation for HF symptom } \\
\hline Received vs none & $1.01(0.97$ to 1.05$)$ & 0.667 & 1.06 (1.00 to 1.12$)$ & 0.060 \\
\hline
\end{tabular}

HF, heart failure; IMD, Index of Multiple Deprivation; NA, not applicable.

guidelines, but European and US guidelines make similar recommendations. ${ }^{521}$ The association between initial management of $\mathrm{HF}$ and patient outcomes is likely to be applicable to many health systems, particularly those where primary care acts as a gatekeeper to specialist services.

\section{Implications for policy and practice}

Diagnosing $\mathrm{HF}$ in primary care is a significant challenge. ${ }^{3} \mathrm{HF}$ manifests in different ways, affecting patients' health-seeking behaviour. From the GP's perspective, difficulties include the non-specificity of HF symptoms, confusion with respiratory conditions, ${ }^{19}$ limited time, 
limited access to investigations, low confidence in interpretation of investigation results, ${ }^{3}$ lack of knowledge of clinical guidelines and perceived information overload. Keeping up to date is a major task. ${ }^{22}$

The lower hazard of admission for patients placed on an appropriate NICE-recommended pathway is encouraging and provides real-world evidence to support NICE guidelines on managing HF. Nevertheless, our results suggest that NICE guidelines (currently being updated) are not followed in primary care for the vast majority of patients who go on to be diagnosed with $\mathrm{HF}$, with a significantly increased associated risk of emergency hospitalisation. Future revisions to quality improvement programmes for $\mathrm{HF}$ - such as the Quality and Outcomes Framework in England-should focus on supporting health professionals to make a timely diagnosis and place patients on a suitable care pathway early in the course of their illness. This would also require the NHS making diagnostic services—such as echocardiography-more widely available, and also through greater support for GPs, for example, through specialist heart nurses working in community settings.

Acknowledgements The Dr Foster Unit at Imperial is affiliated with the National Institute of Health Research (NIHR) Imperial Patient Safety Translational Research Centre. The NIHR Imperial Patient Safety Translational Research Centre is a partnership between the Imperial College Healthcare NHS Trust and Imperial College London. The Dr Foster Unit at Imperial College is grateful for support from the NIHR Biomedical Research Centre funding scheme and the NIHR Collaboration for Leadership in Applied Health Research and Care. The views and opinions expressed herein are those of the authors and do not necessarily reflect those of the NHS, the NIHR, MRC, CCF, NETSCC, the HSR programme or the Department of Health.

Contributors $A B, D K$ and $B H$ conceived and designed this study. $A B$ and $D K$ prepared the data. DK carried out the analysis, overseen by $\mathrm{AB}$ and $\mathrm{BH}$. All authors took part in interpreting the data for this study. All authors commented on and helped to revise drafts of this paper. All authors have approved the final version. $A B$ is the guarantor.

Funding The Dr Foster Unit at Imperial College London is partially funded by a grant from Dr Foster, a private healthcare information company, and authors AB and PPA receive funding from the National Institute of Health Research (NIHR) Imperial Patient Safety Translational Research Centre (PSTRC). The Dr Foster Unit at Imperial College London is partly funded by research grants from NIHR's Health Services and Delivery Research programme. MRC's salary is supported by the NIHR Cardiovascular Biomedical Research Unit at the Royal Brompton Hospital, London.

Competing interests $A B, D K$ and PPA had financial support from Dr Foster for the submitted work. FAM and BH are both general practitioners working in the NHS.

Patient consent Not required.

Ethics approval We have approval from the Secretary of State and the Health Research Authority under Regulation 5 of the Health Service (Control of Patient Information) Regulations 2002 to hold confidential data and analyse them for research purposes (CAG ref $15 / \mathrm{CAG} / 0005$ ). We have approval to use them for research and measuring quality of delivery of healthcare from the London - South East Ethics Committee (REC ref 15/L0/0824). The CPRD Group has obtained ethical approval from a National Research Ethics Service Committee (NRES) for all purely observational research using anonymised CPRD data. This study has been carried out as part of the work approved by their Independent Scientific Advisory Committee (ISAC) with protocol number 16_003RAR.

Provenance and peer review Not commissioned; internally peer reviewed.

Data sharing statement Due to information governance rules applicable to CPRD, no patient-level data are available for sharing.

Open access This is an open access article distributed in accordance with the Creative Commons Attribution Non Commercial (CC BY-NC 4.0) license, which permits others to distribute, remix, adapt, build upon this work non-commercially, and license their derivative works on different terms, provided the original work is properly cited, appropriate credit is given, any changes made indicated, and the use is non-commercial. See: http://creativecommons.org/licenses/by-nc/4.0

\section{REFERENCES}

1. GBD 2015 Disease and Injury Incidence and Prevalence Collaborators. Global, regional, and national incidence, prevalence, and years lived with disability for 310 diseases and injuries, 19902015: a systematic analysis for the Global Burden of Disease Study 2015. Lancet 2016;388:1545-602.

2. Bhatnagar $\mathrm{P}$, Wickramasinghe K, Williams J, et al. The epidemiology of cardiovascular disease in the UK 2014. Heart 2015;101:1182-9.

3. Smeets M, Van Roy S, Aertgeerts B, et al. Improving care for heart failure patients in primary care, GPs' perceptions: a qualitative evidence synthesis. BMJ Open 2016;6:e013459.

4. Koudstaal S, Pujades-Rodriguez M, Denaxas S, et al. Prognostic burden of heart failure recorded in primary care, acute hospital admissions, or both: a population-based linked electronic health record cohort study in 2.1 million people. Eur $J$ Heart Fail 2017;19:1119-27.

5. Ponikowski P, Voors AA, Anker SD. ESC Guidelines for the diagnosis and treatment of acute and chronic heart failure: the Task Force for the diagnosis and treatment of acute and chronic heart failure of the European Society of Cardiology (ESC). Eur Heart $J$ 2016;37:2129-200.

6. National Institute of Health and Care Excellence. Chronic heart failure: national clinical guideline for diagnosis and management in primary and secondary care. National Clinical Guideline Centre, 2010.

7. Scottish Intercollegiate Guidelines Network. SIGN 147 Management of chronic heart failure: a national clinical guideline. SIGN, 2016.

8. Cleland JG, Cohen-Solal A, Aguilar JC, et al. Management of heart failure in primary care (the IMPROVEMENT of Heart Failure Programme): an international survey. Lancet 2002;360:1631-9.

9. Bottle A, Kim D, Aylin P, et al. Routes to diagnosis of heart failure: observational study using linked data in England. Heart 2018;104:600-5.

10. Hawkins NM, Scholes S, Bajekal M, et al. Community care in England: reducing socioeconomic inequalities in heart failure. Circulation 2012;126:1050-7.

11. Khan NF, Perera R, Harper S, et al. Adaptation and validation of the Charlson Index for Read/OXMIS coded databases. BMC Fam Pract 2010;11:11:1

12. Herrett E, Gallagher AM, Bhaskaran K, et al. Data resource profile: Clinical Practice Research Datalink (CPRD). Int J Epidemiol 2015;44:827-36.

13. Chaudhry Z, Mannan F, Gibson-White A, et al. Outputs and growth of primary care databases in the United Kingdom: bibliometric analysis. $J$ Innov Health Inform 2017:24:284-90.

14. Burns EM, Rigby E, Mamidanna R, et al. Systematic review of discharge coding accuracy. J Public Health 2012;34:138-48.

15. Deaton $\mathrm{C}$, Benson J. Time for correct diagnosis and categorisation of heart failure in primary care. Br J Gen Pract 2016;66:554-5.

16. Jordan K, Porcheret M, Croft P. Quality of morbidity coding in general practice computerized medical records: a systematic review. Fam Pract 2004;21:396-412.

17. Delepaul B, Robin G, Delmas C, et al. Who are patients classified within the new terminology of heart failure from the 2016 ESC guidelines? ESC Heart Fail 2017;4:99-104.

18. Tanaka S, Sanuki Y, Ozumi K, et al. Heart failure with preserved vs reduced ejection fraction following cardiac rehabilitation: impact of endothelial function. Heart Vessels 2018;33:886-92.

19. van Riet EES, Hoes AW, Limburg A. Prevalence of unrecognized heart failure in older persons with shortness of breath on exertion: Unrecognized HF in older persons with shortness of breath on exertion. Eur J Heart Fail 2014;16:772-7.

20. Dahlstrom U, Hakansson J, Swedberg K, et al. Adequacy of diagnosis and treatment of chronic heart failure in primary health care in Sweden. Eur J Heart Fail 2009;11:92-8.

21. Yancy CW, Jessup M, Bozkurt B, et al. 2017 ACC/AHA/HFSA Focused Update of the 2013 ACCF/AHA guideline for the management of heart failure: a report of the American College of Cardiology/American Heart Association Task Force on Clinical Practice Guidelines and the Heart Failure Society of America. Circulation 2017;136:e137-61.

22. Alper BS, Hand JA, Elliott SG, et al. How much effort is needed to keep up with the literature relevant for primary care? J Med Libr Assoc 2004;92:429-37. 